\section{What TEM Camera Should I Choose?}

Michael Bode, Michael Wibbelt, ${ }^{*}$ and Christoph Huelk*

Soft Imaging system Corp. ${ }^{*}$ Soft Imaging System GmbH mb@soft-imaging.com

Image acquisition in a TEM requires the conversion of the electron image into a photon image and the acquisition of this photon image with an imaging device. There are two principal techniques to do so: a fiber-coupled camera and a lens-coupled camera. Both have advantages and disadvantages. Please be aware that we (Soft Imaging System Corp) produce both types of cameras. There are also two principle camera positions on a TEM, and the choice of position must be taken into account for any decision. The positions are: side-mount (camera mounted on $35 \mathrm{~mm}$ port above the viewing chamber), and bottom-mount (also known as on-axis mount, camera mounted below the viewing chamber).

Side-mount: If you picture the path of the electrons, they essentially form a cone of illumination below the specimen. At any point of this cone an image can be formed by intercepting the electron beam with a phosphor screen. The resolution of this image is ultimately determined by the resolution of the phosphor screen, which is on the order of 10 microns, depending on phosphor and acceleration voltage.

Lens coupled systems: In lens-coupled systems the electron image is converted to a photon image using a phosphor screen or YAG crystal. The resulting image is then observed through a camera-lens system similar to a normal optical camera.

\section{Advantages:}

Less expensive

Larger selection of cameras

Disadvantages:

Lens distortions (A lens-coupled system typically needs

extremely short focal length lens in order to stay small)

Lower sensitivity (multiple glass/vacuum interfaces)

Larger system

Require periodic re-focusing, unless single port system is used.

Lens-coupled systems are more frequently used for side-mount cameras, where the narrow confinement within the column and the necessity to remove the device from the beam for normal work place limitations on the physical size of the device inside the column. In most cameras, the phosphor screen is inside the column, while the camera sits outside the vacuum. Through a prism or mirror, the camera observes the backside of the phosphor. Custom-made optics are normally used to eliminate distortions. A big advantage of side-mounted cameras is their large field of view, which equals or surpasses that of film. This makes this type of camera ideal for applications such as pathology or other medical applications. The image size of these cameras varies from TV $(640 \times 480)$ to $4000 \times 2500$ (Morada).

Fiber-coupled systems: In these systems the electron image is converted to a photon image using a phosphor screen or YAG crystal. The phosphor is placed in direct contact with a fiber-optic bundle, whose other end is connected to a CCD. The photons are directed directly to the CCD cells through the fibers without a lens. Advantages:

Higher sensitivity

No refocus necessary

Smaller system
No distortion

Disadvantages:

More expensive (especially if a tapered fiber is required)

"chicken-wire" (Moiré pattern due to fiber bundle periodicity)

Fiber-coupled cameras are more frequently used at the bottom-mount position, where higher sensitivity is required. These cameras often also use large, full-frame CCDs, which require external shutters and are read out slowly to avoid read-out noise. Camera sizes range from $1 \mathrm{k} \times 1 \mathrm{k}$ to $4 \mathrm{k} \times 4 \mathrm{k}$. These cameras usually require software that removes the "chicken-wire" effect due to an interference between the CCD and the fiber-bundle periodicities. As these cameras are mounted below the film camera, their field of view is normally smaller than a film plate. This, together with their higher resolution, makes them perfect image acquisition tools for high-resolution TEM.

\section{Which camera should you choose?}

This is one of the most frequently asked questions. The best way to answer this is to look at the normal use of the microscope. If you normally take images on negatives and look at images developed from the entire negative, a side-mount camera will do a good job. There are some high-resolution cameras available (up to $11 \mathrm{Mpixel)}$ that will provide near-film quality at a similar field of view.

If, on the other hand, you find yourself developing your negatives and then spend hours in the darkroom trying to magnify and print tiny areas of a negative, a bottom-mount camera is a better choice. Here you need to weigh both field of view and camera speed. The size of the viewing area is of course important, but also the frame rate: cameras with a frame rate of less than about 8 frames per second require a normal use of the microscope (working in the dark and focusing through binoculars), while higher frame rates allow working on the computer screen. This reduces fatigue and can also be used as a teaching tool.

If your microscope is used by a variety of users, it is usually possible to attach both cameras to a TEM and have the benefits of both. In those cases you should make sure that both cameras can be controlled from the same computer to avoid redundancy and cost.

\section{Software}

Since almost all modern cameras are digital, they are controlled through a computer. This allows further simplification and streamlining through the use of processing, archiving and collaborative tools.

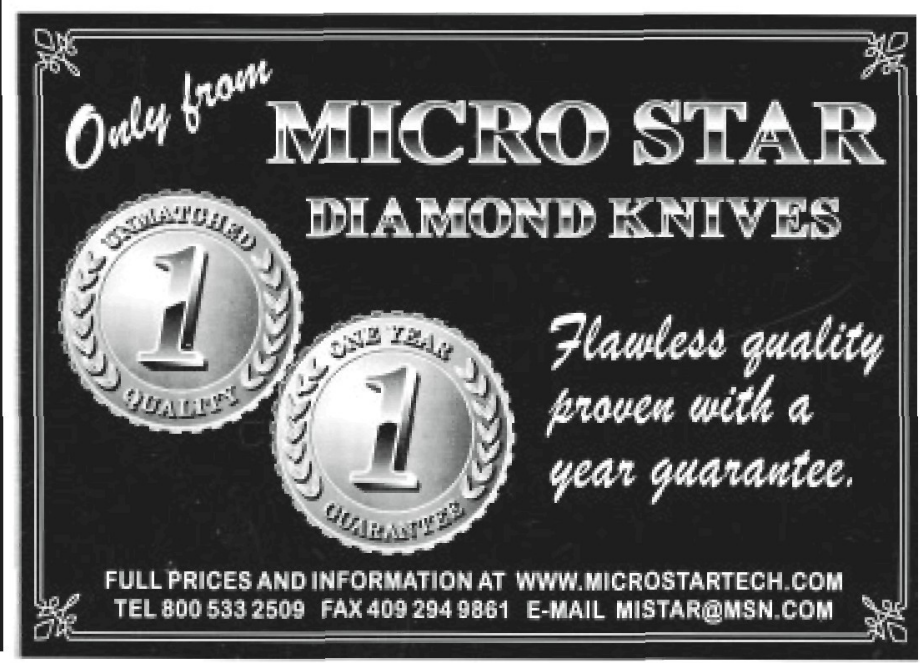




\section{TUR N I N G VISION S I N T O REALITIES}

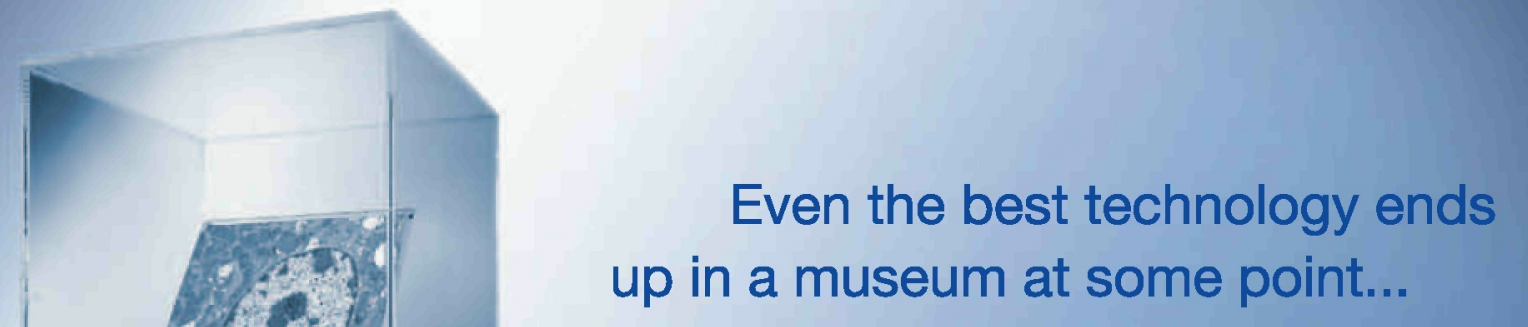

With the release of Soft Imaging System's new TEM side-mounted camera MORADA, it is time to say good-bye to an old and trusty friend who has been with us since the beginning of electron microscopy: the photo plate.

Stop wasting time working in the darkroom, start saving time by electronic reporting within seconds of acquisition. The environment will thank you, too. And you can do that while maintaining or even improving on the image quality you are used to from your photo plate. MORADA is the first sidemounted camera whose features surpass film: the field of view is approximately twice that of film and it shows the same fine detail as film with the same field of view.

More details about the MORADA can be found on our web site:

wwwsoft-imaging.net/TEM

\section{Morada}

11 MegaPlxel side-mounted TEM camera

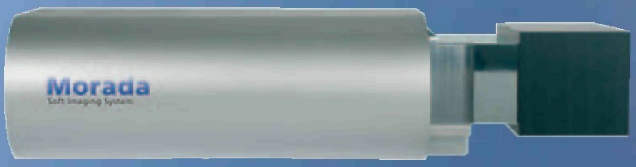

For detalled information please contact:

Soft Imaging System

info.de@soft-imaging.net

www.soft-imaging.net/tem

North America: (888) FIND SIS +1 (303) 234-9270

Europe: +49 (251) 79800-0

Asia | Pacific: $+60(3) 8313-1400$

\section{Soft Imaging System

